D.S. Souza https://orcid.org/0000-0002-0827-7931 S.G.N.S.Yang https://orcid.org/0000-0002-0750-2607 A.C.A. Alves

https://orcid org/0000-0001-9886-3813 R.M. Pontes

\section{Perfil hematológico e bioquímico sérico de capivaras (Hydrochoerus hydrochaeris) de vida livre nos biomas Mata Atlântica e Caatinga}

[Hematological and serum biochemical profile of free-living capybaras (Hydrochoerus hydrochaeris) in Atlantic Forest and Caatinga biomes]

D.S. Souza, S.G.N.S. Yang, A.C.A. Alves, R.M. Pontes, C.C.D. Carvalho, P.C. Soares, J.B. Oliveira*

Universidade Federal Rural de Pernambuco - Recife, PE

\title{
RESUMO
}

Devido à ausência de estudos sobre capivaras na região Nordeste do Brasil, o objetivo deste estudo foi avaliar a sanidade desses roedores de vida livre em três áreas dos biomas Mata Atlântica (2) e Caatinga (1) do estado de Pernambuco, por meio da determinação de parâmetros da hematologia e bioquímica sérica. De novembro de 2016 a dezembro de 2017, foram capturados 21 animais, dos quais foram coletadas amostras de sangue para avaliação hematológica (eritrograma, leucograma e plaquetometria) e bioquímica sérica (atividade enzimática, perfil proteico, energético e mineral). A maioria dos parâmetros esteve dentro dos valores de normalidade para a espécie, embora alguns apresentassem diferenças estatisticamente significativas de acordo com a área de estudo (hemoglobina, hematócrito, VCM, CHCM, eosinófilos, fosfatase alcalina, proteína total, albumina, ácido úrico, creatinina, lactato, sódio e magnésio) e o sexo dos animais (ácido úrico). Os parâmetros obtidos são apresentados como referência e atestam a sanidade e o bom estado nutricional de populações de capivaras nos biomas Mata Atlântica e Caatinga da região Nordeste do Brasil. As informações aportadas neste estudo pioneiro na região Nordeste contribuem para aumentar o conhecimento sobre a ecofisiologia e a conservação in situ de capivaras.

Palavras-chave: roedores, saúde animal, sangue, conservação in situ

\begin{abstract}
Due to the lack of studies about capybaras in the northeast region of Brazil, the objective of this study was to evaluate the health status of free-ranging capybaras in three areas of Atlantic Forest (2) and Caatinga (1) biomes in Pernambuco state, through the determination of hematological and serum biochemical parameters. From November 2016 to December 2017, 21 animals were captured and blood samples were collected for the hematological (erythrogram, leukogram and platelet counts) and serum biochemistry (enzymatic activity, protein, energy and mineral profile) evaluation. Hematological and serum biochemical parameters were within the normal range for the species, but some presented statistically significant variations according to the study area (hemoglobin, hematocrit, MCV, MCHC, eosinophils count, alkaline phosphatase, total proteins, albumin, uric acid, creatinine, lactate, sodium and magnesium) and sex of the animals (uric acid). The parameters obtained are presented as reference and attest to the health and good nutritional status of populations of capybaras in the Atlantic Forest and Caatinga biomes of northeastern Brazil. The information provided in this pioneering study in the northeast region contributes to increased knowledge about the ecophysiology and in situ conservation of capybaras.
\end{abstract}

Keywords: rodents, animal health, blood, in situ conservation

Recebido em 27 de novembro de 2018

Aceito em 27 de março de 2019

*Autor para correspondência (corresponding author)

E-mail: bianque01@yahoo.com.br 


\section{INTRODUÇÃO}

A capivara (Hydrochoerus hydrochaeris Linnaeus, 1766) é considerada o maior roedor vivo do mundo, sendo uma espécie nativa da América do Sul, com populações ocorrendo em ambientes como matas ciliares e áreas abertas ou campos (Ferraz e Verdade, 2001). Esse roedor se caracteriza por sua plasticidade alimentar, adaptação a ambientes antropizados e resistência a doenças (Jiménez, 1995; Nogueira e NogueiraFilho, 1996), e é hospedeiro de uma ampla variedade de patógenos, alguns com potencial zoonótico (Truppel, 2009; Di-Chiacchio et al., 2014). Com o desequilíbrio ecológico e alterações em seu habitat natural, as capivaras invadem ambientes urbanos e áreas de lavouras, o que vem sendo observado em Pernambuco e em outros estados da região Nordeste do Brasil, onde não existem estudos sobre o estado de saúde desses mamíferos.

Devido à natureza dinâmica dos parâmetros fisiológicos dos animais silvestres, o contexto ecológico e ambiental deve ser levado em consideração na avaliação do estado de saúde desses animais (Eberhardt et al., 2015). O conhecimento sobre os parâmetros hematológicos e bioquímicos pode fornecer informações valiosas sobre a história natural e o estado de saúde e nutricional de espécies de vertebrados silvestres (Di-Chiacchio et al., 2014; Eberhardt et al., 2015, 2017), contribuindo para o manejo e a conservação desses animais e dos ecossistemas onde vivem. As informações sobre os parâmetros fisiológicos de capivaras são oriundas das regiões Sudeste, Centro-Oeste e Sul do Brasil (Muñoz e Montoya, 2001; Heijden et al., 2003; Madella et al., 2006; Corredor-Matus e Rodríguez-Pulido, 2010; Di-Chiacchio et al., 2014) e inexistentes nos biomas Mata Atlântica e Caatinga da região Nordeste.

De acordo com Quadros (2017) e Di-Chiacchio et al. (2014), a desnutrição, principalmente na época seca, é um dos principais problemas que afetam capivaras em vida livre, influenciando alguns parâmetros hematológicos e bioquímicos. Os objetivos deste estudo foram: (1) determinar os valores hematológicos (eritrograma, leucograma e plaquetometria) e bioquímicos séricos (atividade enzimática, perfil proteico, metabólico e mineral) de capivaras em vida livre na Mata Atlântica e na Caatinga e (2) testar a influência de fatores ambientais (biomas), do animal (sexo) e do parasitismo (parasitos gastrointestinais e carrapatos) sobre os parâmetros avaliados. As informações obtidas contribuirão para a conservação in situ, a partir do conhecimento sobre a ecofisiologia e o estado de saúde desses animais na região Nordeste do Brasil.

\section{MATERIAL E MÉTODOS}

De novembro de 2016 a dezembro de 2017, após autorização do Sisbio ( $\mathrm{N}^{0}$ 53750-3) e licença da Comissão de Ética para o Uso de Animais da Universidade Federal Rural de Pernambuco (CEUA/UFRPE $\mathrm{N}^{\mathrm{o}}$ 073/2016), foram capturados 21 animais oriundos de três áreas nos biomas Mata Atlântica (áreas 1 e 2) e Caatinga (área 3), no estado de Pernambuco:

Área 1- Parque Estadual Dois Irmãos, unidade de conservação da Mata Atlântica com 1.157,72ha, situada no município do Recife $\left(08^{\circ} 03^{\prime} 14\right.$ 'S, 3452'52" W). Segundo a classificação de Köppen, o clima da região é do tipo As' (quente e úmido), com temperatura média anual de $25,8^{\circ} \mathrm{C}$, precipitação média anual de aproximadamente $2.460 \mathrm{~mm}$, e a vegetação é composta por Floresta Estacional Perenifólia Costeira ou Floresta Ombrófila Densa. Nessa área, foi identificado um único grupo de 12 indivíduos que vivem às margens de um açude, dos quais foram capturados 10 animais (seis machos e quatro fêmeas).

Área 2 - Estação Ecológica do Tapacurá (EET), localizada no município de São Lourenço da Mata $\left(08^{\circ} 02^{\prime} 39^{\prime}\right.$ 'S e $\left.35^{\circ} 12^{\prime} 05^{\prime} \mathrm{W}\right)$, com uma área de 776 ha, constituída por três unidades de conservação de proteção integral da Mata Atlântica. É classificada como Floresta Estacional Caducifólia (Mata Atlântica seca). O clima da região é do tipo As' (quente e úmido), com precipitação média anual de $1.900 \mathrm{~mm}$ e temperatura média anual de $27^{\circ} \mathrm{C}$. De um grupo de 16 animais que vivem às margens do lago da barragem, foram capturados seis (quatro fêmeas adultas, uma fêmea e um macho juvenis).

Área 3 - Reserva Particular do Patrimônio Natural (RPPN) Preservação Chácara Paraíso (10 ha), localizada no município de Chã Grande $\left(8^{\circ} 14^{\prime} 18^{\prime \prime}\right.$ S e $\left.35^{\circ} 27^{\prime} 42^{\prime \prime} \mathrm{W}\right)$. Apresenta vegetação predominantemente do tipo Floresta 
Subperenifólia, com partes de Caatinga Hipoxerófila e o clima é do tipo As', com precipitação média anual de $1.309,9 \mathrm{~mm}$ e temperatura média de $21,9^{\circ} \mathrm{C}$. O grupo era constituído por 15 indivíduos que vivem às margens de um lago, dos quais cinco adultos foram capturados (quatro fêmeas e um macho).

Após localizados os grupos e identificadas suas respectivas áreas de uso, os animais foram condicionados, por meio de ceva, a entrar em um cercado construído em local sombreado, utilizando a vegetação natural, com estacas de madeira e telas de alambrado. Quando capturados, os animais permaneceram em jejum hídrico e alimentar (entre 12 e 15 horas) antes da contenção química. Uma vez no brete, foi realizado o protocolo de contenção química que consistiu da associação de diazepam $(0,2 \mathrm{mg} / \mathrm{kg})$, cloridrato de cetamina $(7,0 \mathrm{mg} / \mathrm{kg})$ e cloridrato de xilazina $(0,4 \mathrm{mg} / \mathrm{kg})$, aplicados por via intramuscular. Após a contenção química, foram realizados os procedimentos de coleta de amostras biológicas, marcação, biometria, sexagem, aferição da temperatura retal e das frequências cardíaca e respiratória, além da observação da coloração das mucosas oral e ocular. Os animais foram manejados individualmente e a soltura ocorreu somente após a plena recuperação.

De cada animal foram coletados entre 10 e $12 \mathrm{~mL}$ de sangue, por meio da punção das veias femorais ou cefálicas. Desse total, 2,0mL foram armazenados em tubos de MiniCollect ${ }^{\circledR} \mathrm{CE}$ contendo EDTA (ácido dietilenodiaminotetracético), e o restante de cada amostra foi depositado em tubo com gel separador. As amostras foram acondicionadas em caixa isotérmica refrigerada e enviadas ao laboratório no mesmo dia da coleta.

Para a determinação dos valores de leucócitos $\left(/ \mathrm{mm}^{3}\right)$, eritrócitos $\left(10^{6} / \mathrm{mm}^{3}\right)$, hemoglobina $(\mathrm{Hb})$ $(\mathrm{g} / \mathrm{dL})$, hematócrito $(\mathrm{Ht}) \quad(\%)$, volume corpuscular médio (VCM) (fL), concentração de hemoglobina corpuscular média $(\mathrm{CHCM})(\mathrm{g} / \mathrm{dL})$ e plaquetas $\left(/ \mathrm{mm}^{3}\right)$, as amostras foram processadas em analisador hematológico automático AbcVet-Scil (Illinois, USA). O princípio do equipamento utiliza a citometria de fluxo para determinação do número de partículas (eritrócitos, leucócitos e plaquetas), diferenciando-as por impedância, e a espectrofotometria para determinação da hemoglobina.

Foram confeccionados esfregaços sanguíneos corados pelo método panótico para observação morfológica das células vermelhas e contagem diferencial das células brancas. No soro sanguíneo, foram determinados, por meio de analisador bioquímico automático Labmax $240^{\circledR}$ : proteína total (PT) (g/dL), albumina (AL) (g/dL), globulina $(\mathrm{g} / \mathrm{dL})$, ácido úrico $(\mathrm{mg} / \mathrm{dL})$, ureia $(\mathrm{U})$ (mg/dL), creatinina (C) (mg/dL), alanina aminotransferase (ALT) (U/L), aspartato aminotransferase (AST) (U/L), gamaglutamiltransferase (GGT) (U/L), fosfatase alcalina (Falc) (U/L), colesterol (mg/dL), triglicerídeos (mg/dL), HDL $(\mathrm{mg} / \mathrm{dL})$, frutosamina $(\mathrm{mmol} / \mathrm{L})$, lactato $(\mathrm{mg} / \mathrm{dL})$ e os minerais $\mathrm{Ca}(\mathrm{mg} / \mathrm{dL}), \mathrm{P}(\mathrm{mg} / \mathrm{dL}), \mathrm{Mg}(\mathrm{mg} / \mathrm{dL}), \mathrm{K}$ $(\mathrm{mEq} / \mathrm{L}), \mathrm{Na}(\mathrm{mEq} / \mathrm{L})$ e $\mathrm{Cl}(\mathrm{mEq} / \mathrm{L})$.

Para a análise quanto à distribuição normal do conjunto de dados, utilizou-se o teste de Kolmogorov-Smirnov. As variáveis que não atenderam às premissas de normalidade foram submetidas à transformação logarítmica ou da raiz quadrada de $x+1$. Os dados com distribuição normal foram submetidos à análise de variância (ANOVA), utilizando-se o procedimento GLM do SAS - Statistical Analysis System (SAS..., 2009); os dados não paramétricos foram submetidos ao teste de Kruskal-Wallis, para dois grupos independentes (áreas de estudo), e ao teste de Mann-Whitney, para comparação de medianas com dois grupos independentes (sexo). $\mathrm{Na}$ ANOVA, o contraste de médias foi realizado pela diferença mínima significativa (d.m.s.) do teste de Student-Newman-Keuls. Para todas as análises, foi considerado o nível de significância ( $p$ ) de 5\%. Os dados foram caracterizados por dispersão de frequências, utilizando-se as seguintes medidas de tendência central: média, desvio-padrão, mediana, percentil de $25 \%$ e percentil de $75 \%$.

\section{RESULTADOS}

No total, foram capturados 21 animais, sendo 11 adultos e 10 juvenis de ambos os sexos (13 fêmeas e oito machos). A média de peso dos animais foi $32,12 \pm 15,79 \mathrm{~kg}(25,71 \pm 14,18 \mathrm{~kg}$ para machos e $38,53 \pm 17,40 \mathrm{~kg}$ para fêmeas). O escore corporal apresentou-se dentro de parâmetros esperados para a espécie. Na avaliação clínica, os 
animais apresentaram mucosas levemente hipocoradas e dentição saudável; em dois animais, foram observadas lesões de pele sem sinal de infecção. Os parâmetros hematológicos se mostraram dentro dos valores de referência para a espécie, de acordo com o Sistema
Internacional de Informação de Espécies (ISIS, 2013) (Tab. 1), enquanto os parâmetros de atividade enzimática (FA e AST), perfil proteico (albumina e ureia) e mineral $(\mathrm{Na})$ se mostraram levemente alterados em relação ao ISIS (2013) (Tab. 1).

Tabela 1. Parâmetros hematológicos e bioquímicos séricos de capivaras (Hydrochoerus hydrochaeris) de vida livre nos biomas Mata Atlântica e Caatinga do Nordeste do Brasil.

\begin{tabular}{|c|c|c|}
\hline Parâmetros/Unidades & Média \pm Desvio-Padrão & Valores de Referência $^{* *}$ \\
\hline \multicolumn{3}{|c|}{ Eritrograma } \\
\hline $\mathrm{He}\left(\mathrm{x} 10^{6} / \mathrm{mm}^{3}\right)$ & $2,71 \pm 0,37$ & $2,18-4,83$ \\
\hline $\mathrm{Hb}(\mathrm{g} \%)$ & $10,90 \pm 1,88$ & $9,8-18,00$ \\
\hline $\mathrm{Ht}(\%)$ & $33,03 \pm 6,32$ & $29,6-56,40$ \\
\hline VCM (fL) & $124,13 \pm 19,93$ & $88,0-157,80$ \\
\hline CHCM (g/dL) & $32,59 \pm 1,62$ & $29,8-38,20$ \\
\hline \multicolumn{3}{|c|}{ Leucograma } \\
\hline Leucócitos (x/mm³)x1000 & $5,85 \pm 2,05$ & $2,93-13,41$ \\
\hline Segmentados $\left(\mathrm{mm}^{3}\right) \times 1000$ & $3,56 \pm 1,43$ & $0,09-8,70$ \\
\hline Eosinófilos $\left(\mathrm{mm}^{3}\right)$ & $415,86 \pm 439,92$ & $0-534$ \\
\hline Basófilos $^{*}\left(\mathrm{~mm}^{3}\right)$ & $0,0(0,0 ; 113,0)$ & --- \\
\hline Monócitos* $\left(\mathrm{mm}^{3}\right)$ & $465,5(202,1 ; 1326,5)$ & $39-1071$ \\
\hline Linfócitos $\left(\mathrm{mm}^{3}\right)$ & $1307,74 \pm 656,16$ & $500-5730$ \\
\hline \multicolumn{3}{|c|}{ Plaquetometria } \\
\hline Plaquetas ${ }^{*}(\mathrm{x} 1000)$ & $215,2(110,5 ; 692,0)$ & $85-429$ \\
\hline \multicolumn{3}{|c|}{ Atividade Enzimática } \\
\hline ALT (U/L) & $66,85 \pm 29,81$ & $15-69$ \\
\hline AST (U/L) & $209,43 \pm 171,66$ & $12-102$ \\
\hline $\mathrm{GGT}^{*}(\mathrm{U} / \mathrm{L})$ & $0,5(0,0 ; 5,0)$ & $0-9$ \\
\hline FA (U/L) & $179,58 \pm 137,39$ & $58-1074$ \\
\hline \multicolumn{3}{|c|}{ Perfil Proteico } \\
\hline PT $(g / d L)$ & $6,53 \pm 0,57$ & $4,5-7,70$ \\
\hline Albumina (g/dL) & $4,62 \pm 0,46$ & $1,5-4,10$ \\
\hline Globulina (g/dL) & $1,91 \pm 0,19$ & $1,5-4,90$ \\
\hline $\mathrm{A}: \mathrm{G}$ & $2,43 \pm 0,25$ & ----- \\
\hline Ácido úrico ${ }^{*}$ (mg/dL) & $0,8(0,0 ; 1,1)$ & $0-2,86$ \\
\hline Ureia $(\mathrm{mg} / \mathrm{dL})$ & $32,42 \pm 7,58$ & $5,04-24,36$ \\
\hline Creatinina (mg/dL) & $1,51 \pm 0,57$ & $0,89-2,77$ \\
\hline Frutosamina (mg/dL) & $248,03 \pm 36,47$ & ----- \\
\hline \multicolumn{3}{|c|}{ Perfil Energético } \\
\hline Colesterol (mg/dL) & $41,72 \pm 11,07$ & $35,95-124,50$ \\
\hline Triglicerídeos* $(\mathrm{mg} / \mathrm{dL})$ & $60,5(31,7 ; 159,1)$ & $0-237,18$ \\
\hline $\mathrm{HDL}(\mathrm{mg} / \mathrm{dL})$ & $3,50 \pm 0,80$ & ----- \\
\hline Lactato $^{*}(\mathrm{mg} / \mathrm{dL})$ & $24,2(10,0 ; 81,4)$ & ---- \\
\hline \multicolumn{3}{|c|}{ Perfil Mineral } \\
\hline $\mathrm{Ca}(\mathrm{mg} / \mathrm{dL})$ & $10,37 \pm 0,91$ & $9,73-13,15$ \\
\hline $\mathrm{P}(\mathrm{mg} / \mathrm{dL})$ & $5,82 \pm 0,53$ & $2,883-10,76$ \\
\hline $\mathrm{Ca}: \mathrm{P}$ & $1,81 \pm 0,26$ & $1,0-3,60$ \\
\hline $\mathrm{Mg}(\mathrm{mg} / \mathrm{dL})$ & $2,80 \pm 0,22$ & ----- \\
\hline $\mathrm{Na}(\mathrm{mg} / \mathrm{dL})$ & $172,52 \pm 26,13$ & $127-145$ \\
\hline $\mathrm{K}(\mathrm{mg} / \mathrm{dL})$ & $4,70 \pm 1,76$ & $3,7-7,50$ \\
\hline $\mathrm{Cl}(\mathrm{mg} / \mathrm{dL})$ & $90,19 \pm 4,17$ & ----- \\
\hline
\end{tabular}

*Variáveis com dados não paramétricos - Mediana (P25; P75); **ISIS (2013). 
Em relação à área de origem dos animais, houve diferença significativa nos valores de $\mathrm{Hb}$ $(\mathrm{P}=0,0042)$, Ht $(\mathrm{P}=0,0231)$, VCM $(\mathrm{P}<0,001)$, CHCM $(\mathrm{P}=0,0300)$ e eosinófilos $(\mathrm{P}=0,0041), \mathrm{FA}$ $(\mathrm{P}=0,0276)$, proteína total $(\mathrm{P}=0,0177)$, albumina $(\mathrm{P}=0,0163)$, ácido úrico $(\mathrm{P}=0,0032)$, creatinina $(\mathrm{P}<0,001)$, lactato $(\mathrm{P}=0,0081), \mathrm{Mg}(\mathrm{P}=0,0414)$ e $\mathrm{Na}(\mathrm{P}<0,001)$. Na análise quanto ao sexo dos animais, houve diferença estatisticamente significativa para o valor do ácido úrico $(\mathrm{P}=0,0315)$.

Tabela 2. Parâmetros hematológicos de capivaras (Hydrochoerus hydrochaeris) de vida livre nos biomas Mata Atlântica (áreas 1 e 2) e Caatinga (área 3) do Nordeste do Brasil

\begin{tabular}{|c|c|c|c|c|}
\hline \multirow{2}{*}{ Parâmetros/Unidades } & \multicolumn{3}{|c|}{ Áreas } & \multirow{2}{*}{$\begin{array}{l}\text { Valores de } \\
\text { referência*** }\end{array}$} \\
\hline & 1 & 2 & 3 & \\
\hline \multicolumn{5}{|c|}{ Eritrograma } \\
\hline $\mathrm{He}\left(\mathrm{x} 10^{6} / \mathrm{mm}^{3}\right)$ & $2,78 \pm 0,43$ & $2,56 \pm 0,14$ & $2,60 \pm 0,51$ & $2,18-4,83$ \\
\hline $\mathrm{Hb}(\mathrm{g} \%)$ & $11,45 \pm 1,11^{\mathrm{a}}$ & $8,67 \pm 1,16^{\mathrm{b}}$ & $11,84 \pm 2,19^{\mathrm{a}}$ & $9,8-18,0$ \\
\hline $\mathrm{Ht}(\%)$ & $34,76 \pm 6,17^{\mathrm{a}}$ & $25,95 \pm 3,65^{\mathrm{b}}$ & $35,60 \pm 6,07^{\mathrm{a}}$ & $29,6-56,4$ \\
\hline VCM (fL) & $131,49 \pm 13,57^{\mathrm{a}}$ & $99,10 \pm 10,75^{\mathrm{b}}$ & $137,72 \pm 5,76^{\mathrm{a}}$ & $88,0-157,8$ \\
\hline CHCM (\%) & $31,70 \pm 1,32^{\mathrm{a}}$ & $\begin{array}{l}33,87 \pm 0,79^{\mathrm{a}} \\
\text { Leucograma }\end{array}$ & $33,24 \pm 1,83^{\mathrm{ab}}$ & $29,8-38,2$ \\
\hline $\begin{array}{l}\text { Leucócitos }\left(\mathrm{x} / \mathrm{mm}^{3}\right) \\
\text { x1000 }\end{array}$ & $4,99 \pm 1,64$ & $6,88 \pm 1,81$ & $6,48 \pm 2,36$ & $2,93-13,41$ \\
\hline $\begin{array}{l}\text { Segmentados }\left(\mathrm{mm}^{3}\right) \\
\mathrm{x} 1000\end{array}$ & $3,05 \pm 1,36$ & $3,90 \pm 0,97$ & $4,04 \pm 1,67$ & $0,09-8,70$ \\
\hline Eosinófilos $\left(\mathrm{mm}^{3}\right)$ & $162,78 \pm 156,54^{\mathrm{b}}$ & $932,83 \pm 488,44^{\mathrm{a}}$ & $394,60 \pm 261,09^{\mathrm{ab}}$ & $0-534$ \\
\hline Basófilos ${ }^{*}\left(\mathrm{~mm}^{3}\right)$ & $\begin{array}{c}19,0 \\
(0,0 ; 114,0)\end{array}$ & $\begin{array}{c}0,0 \\
(0,0 ; 136,0)\end{array}$ & $\begin{array}{c}0,0 \\
(0,0 ; 0,0)\end{array}$ & --- \\
\hline Monócitos ${ }^{*}\left(\mathrm{~mm}^{3}\right)$ & $\begin{array}{c}384,0 \\
(226,8 ; 913,0)\end{array}$ & $\begin{array}{c}563,0 \\
(408,0 ; 1740,0)\end{array}$ & $\begin{array}{c}531,0 \\
(168,0 ; 848,0)\end{array}$ & $39-1071$ \\
\hline Linfócitos $\left(\mathrm{mm}^{3}\right)$ & $1271,66 \pm 768,37$ & $\begin{array}{l}1262,83 \pm 523,24 \\
\text { Plaquetometria }\end{array}$ & $1554,80 \pm 946,10$ & $500-5730$ \\
\hline Plaquetas*(x1000) & $\begin{array}{c}250,5 \\
(152,0 ; 959,0)\end{array}$ & $\begin{array}{c}157,0 \\
(113,0 ; 350,0)\end{array}$ & $\begin{array}{c}240,7 \\
(108,0 ; 387,6)\end{array}$ & $85-429$ \\
\hline
\end{tabular}

* Variáveis com dados não paramétricos; **ISIS (2013).

$\mathrm{Na}$ Tab. 3, são apresentados os valores de atividade enzimática e perfil proteico, energético e mineral dos animais das três áreas de estudo. A maior parte dos parâmetros se manteve dentro dos valores de referência (ISIS, 2013), embora valores superiores tenham sido observados na AST, na albumina, na globulina, na ureia, no colesterol, nos triglicerídeos e no Na (Tab. 3). Os parâmetros FA, PT, albumina, ácido úrico, creatinina, lactato, $\mathrm{Mg}$ e $\mathrm{Na}$ apresentaram diferenças estatisticamente significativas entre as áreas (Tab. 3). Em relação ao sexo dos animais, os valores do hemograma e da bioquímica sérica
Os valores médios do hemograma dos animais das áreas 1 e 2 (Mata Atlântica) e 3 (Caatinga) se mantiveram dentro do padrão de normalidade (ISIS, 2013) (Tab. 2). Apesar disso, os valores de $\mathrm{Hb}, \mathrm{Ht}$ e VCM dos animais da área 2 (Mata Atlântica) apresentaram resultados inferiores em relação às demais áreas (Tab. 2). Os valores de $\mathrm{Hb}, \mathrm{Ht}, \mathrm{VCM}$ e CHCM apresentaram diferenças estatisticamente significativas entre as áreas (Tab. 2). 
Tabela 3. Atividade enzimática e perfil proteico, energético e mineral de capivaras (Hydrochoerus hydrochaeris) de vida livre nos biomas Mata Atlântica (áreas 1 e 2) e Caatinga (área 3) do Nordeste do Brasil

\begin{tabular}{|c|c|c|c|}
\hline \multirow{2}{*}{ Parâmetros/Unidades } & \multicolumn{3}{|c|}{ Áreas } \\
\hline & 1 & 2 & 3 \\
\hline & \multicolumn{3}{|c|}{ Atividade Enzimática } \\
\hline ALT (U/L) & $68,48 \pm 25,83$ & $54,22 \pm 5,02$ & $65,01 \pm 56,50$ \\
\hline AST (U/L) & $123,80 \pm 79,93$ & $274,95 \pm 277,81$ & $356,32 \pm 262,10$ \\
\hline $\mathrm{GGT}^{*}(\mathrm{U} / \mathrm{L})$ & $0,78(0,0 ; 3,4)$ & $0,65(0,3 ; 6,7)$ & $0,00(0,0 ; 0,9)$ \\
\hline \multirow[t]{2}{*}{ FA (U/L) } & $124,33 \pm 107,98^{\mathrm{b}}$ & $166,23 \pm 119,26^{\mathrm{ab}}$ & $309,52 \pm 164,85^{\mathrm{a}}$ \\
\hline & \multicolumn{3}{|c|}{ Perfil Proteico } \\
\hline PT (g/dL) & $6,21 \pm 0,43^{b}$ & $6,98 \pm 0,38^{\mathrm{a}}$ & $6,74 \pm 0,57^{\mathrm{ab}}$ \\
\hline Albumina (g/dL) & $4,36 \pm 0,38^{\mathrm{b}}$ & $4,96 \pm 0,27^{\mathrm{a}}$ & $4,81 \pm 0,44^{\mathrm{a}}$ \\
\hline Globulina (g/dL) & $1,86 \pm 0,16$ & $2,02 \pm 0,16$ & $1,93 \pm 0,26$ \\
\hline $\mathrm{A}: \mathrm{G}$ & $2,36 \pm 0,27$ & $2,46 \pm 0,17$ & $2,52 \pm 0,36$ \\
\hline Ácido úrico* & $0,32(0,0 ; 0,9)^{\mathrm{b}}$ & $0,97(0,8 ; 1,3)^{\mathrm{a}}$ & $0,91(0,76 ; 1,1)^{\mathrm{a}}$ \\
\hline Ureia $(\mathrm{mg} / \mathrm{dL})$ & $32,33 \pm 7,56$ & $38,43 \pm 6,63$ & $26,80 \pm 5,10$ \\
\hline Creatinina $(\mathrm{mg} / \mathrm{dL})$ & $1,08 \pm 0,23$ & $1,75 \pm 0,56^{\mathrm{b}}$ & $2,37 \pm 0,30^{\mathrm{a}}$ \\
\hline \multirow[t]{2}{*}{ Frutosamina } & $256,45 \pm 30,54$ & $257,81 \pm 57,98$ & $217,29 \pm 13,68$ \\
\hline & \multicolumn{3}{|c|}{ Perfil Energético } \\
\hline Colesterol total (mg/dL) & $37,49 \pm 7,24$ & $49,37 \pm 14,38$ & $40,92 \pm 10,70$ \\
\hline Triglicerídeos ${ }^{*}(\mathrm{mg} / \mathrm{dL})$ & $59,95(29,9 ; 112,4)$ & $53,83(33,4 ; 205,8)$ & $62,88(45,8 ; 83,4)$ \\
\hline $\mathrm{HDL}(\mathrm{mg} / \mathrm{dL})$ & $3,58 \pm 0,98$ & $3,09 \pm 0,38$ & $3,71 \pm 0,65$ \\
\hline \multirow{2}{*}{ Lactato ${ }^{*}(\mathrm{mg} / \mathrm{dL})$} & $14,39(10,0 ; 49,01)^{\mathrm{b}}$ & $53,78(22,01 ; 94,5)^{\mathrm{a}}$ & $26,77(24,7 ; 51,4)^{\mathrm{b}}$ \\
\hline & \multicolumn{3}{|c|}{ Perfil Mineral } \\
\hline $\mathrm{Ca}(\mathrm{mg} / \mathrm{dL})$ & $10,52 \pm 0,93$ & $10,65 \pm 0,79$ & $9,68 \pm 0,45$ \\
\hline $\mathrm{P}(\mathrm{mg} / \mathrm{dL})$ & $6,07 \pm 0,51$ & $5,38 \pm 0,96$ & $5,46 \pm 0,45$ \\
\hline $\mathrm{Ca}: \mathrm{P}$ & $1,74 \pm 0,21$ & $2,04 \pm 0,46$ & $1,78 \pm 0,09$ \\
\hline $\operatorname{Mg}(\mathrm{mg} / \mathrm{dL})$ & $2,84 \pm 0,24^{\mathrm{a}}$ & $2,87 \pm 0,12^{\mathrm{a}}$ & $2,56 \pm 0,17^{\mathbf{b}}$ \\
\hline $\mathrm{Na}(\mathrm{mEq} / \mathrm{L})$ & $160,65 \pm 12,69^{\mathrm{b}}$ & $163,03 \pm 16,52^{b}$ & $207,84 \pm 22,62^{\mathrm{a}}$ \\
\hline $\mathrm{K}(\mathrm{mEq} / \mathrm{L})$ & $5,10 \pm 2,24$ & $4,58 \pm 1,08$ & $3,98 \pm 0,24$ \\
\hline $\mathrm{Cl}(\mathrm{mEq} / \mathrm{L})$ & $89,97 \pm 2,18$ & $89,44 \pm 8,37$ & $91,69 \pm 3,16$ \\
\hline
\end{tabular}

*Variáveis com dados não paramétricos.

\section{DISCUSSÃO}

O conhecimento sobre os parâmetros hematológicos e bioquímicos séricos pode fornecer informações valiosas sobre a história natural de vertebrados silvestres (Di-Chiacchio et al., 2014; Eberhardt et al., 2015, 2017), além de indicar a ocorrência de enfermidades infectoparasitárias, metabólicas e nutricionais (Arouca et al., 2000; Muñoz e Montoya, 2001; Carvalho et al., 2013; Di-Chiacchio et al., 2014), entre outras patologias, contribuindo, dessa forma, para o manejo e a conservação in situ e ex situ desses animais e dos ecossistemas onde vivem (Corredor-Matus e Rodríguez-Pulido, 2010).
A avaliação biométrica de animais é um parâmetro importante para o conhecimento do seu estado de saúde. Em animais gregários, como as capivaras, a massa corporal pode determinar a dominância no grupo (Di-Chiacchio et al., 2014). Neste estudo, a diferença na média de peso entre machos e fêmeas pode ser atribuída à quantidade de machos juvenis, que foi maior do que de machos adultos. No estudo realizado por DiChiacchio et al. (2014), em São Paulo, o peso dos animais adultos foi superior (machos $56,5 \pm 12,66 \mathrm{~kg}$ e fêmeas $57,2 \pm 6,30 \mathrm{~kg}$ ) ao dos animais do presente estudo. A qualidade e a abundância de alimentos poderiam explicar essas diferenças (Jiménez, 1995). 
Tabela 4. Parâmetros hematológicos e bioquímicos séricos, de acordo com o sexo, de capivaras (Hydrochoerus hydrochaeris) de vida livre nos biomas Mata Atlântica e Caatinga do Nordeste do Brasil

\begin{tabular}{|c|c|c|c|}
\hline \multirow{2}{*}{ Parâmetros/Unidades } & \multicolumn{2}{|c|}{ Sexo } & \multirow{2}{*}{$\begin{array}{l}\text { Valores de } \\
\text { referência*** }\end{array}$} \\
\hline & Macho & Fêmea & \\
\hline \multicolumn{4}{|c|}{ Eritrograma } \\
\hline $\mathrm{He}\left(\mathrm{x} 10^{6} / \mathrm{mm}^{3}\right)$ & $2,88 \pm 0,44$ & $2,54 \pm 0,31$ & $2,18-4,83$ \\
\hline $\mathrm{Hb}(\mathrm{g} \%)$ & $11,56 \pm 1,99$ & $10,25 \pm 1,77$ & $9,8-18,00$ \\
\hline $\mathrm{Ht}(\%)$ & $35,51 \pm 5,88$ & $30,55 \pm 6,77$ & $29,6-56,40$ \\
\hline VCM (fL) & $125,83 \pm 20,00$ & $122,43 \pm 19,85$ & $88,0-157,80$ \\
\hline $\mathrm{CHCM}(\%)$ & $32,20 \pm 1,69$ & $32,99 \pm 1,55$ & $29,8-38,20$ \\
\hline \multicolumn{4}{|c|}{ Leucograma } \\
\hline Leucócitos $\left(10^{3} / \mathrm{mm}^{3}\right)$ & $5,70 \pm 2,15$ & $6,00 \pm 1,96$ & $2,93-13,410$ \\
\hline Segmentados $\left(10^{3} / \mathrm{mm}^{3}\right)$ & $3,68 \pm 1,65$ & $3,44 \pm 1,21$ & $0,09-8,70$ \\
\hline Eosinófilos $\left(\mathrm{mm}^{3}\right)$ & $322,9 \pm 424,17$ & $508,8 \pm 455,66$ & $0-534$ \\
\hline Basófilos ${ }^{*}\left(\mathrm{~mm}^{3}\right)$ & $0,0(0,0 ; 0,0)$ & $0,00(90,0 ; 136,0)$ & --- \\
\hline Monócitos* ${ }^{*}\left(\mathrm{~mm}^{3}\right)$ & $400,0(168,0 ; 236,3)$ & $531,0(913,0 ; 1740,0)$ & $39-1071$ \\
\hline Linfócitos $\left(\mathrm{mm}^{3}\right)$ & $1186,73 \pm 453,84$ & $1428,75 \pm 858,48$ & $500-5730$ \\
\hline \multicolumn{4}{|c|}{ Plaquetometria } \\
\hline Plaquetas*(x1000) & $212,5(108,0 ; 113,0)$ & $217,8(959,0 ; 425,0)$ & $85-429$ \\
\hline \multicolumn{4}{|c|}{ Atividade Enzimática } \\
\hline $\operatorname{ALT}(\mathrm{U} / \mathrm{L})$ & $80,61 \pm 34,28$ & $53,09 \pm 25,33$ & $15-69$ \\
\hline AST (U/L) & $155,18 \pm 82,63$ & $263,68 \pm 260,69$ & $12-102$ \\
\hline $\mathrm{GGT}^{*}(\mathrm{U} / \mathrm{L})$ & $0,28(0,0 ; 0,0)$ & $0,76(3,4 ; 6,7)$ & $0-9$ \\
\hline $\mathrm{FA}(\mathrm{U} / \mathrm{L})$ & $176,17 \pm 114,28$ & $182,99 \pm 160,49$ & $58-1074$ \\
\hline \multicolumn{4}{|c|}{ Perfil Proteico } \\
\hline PT $(g / d L)$ & $6,41 \pm 0,64$ & $6,65 \pm 0,49$ & $4,5-7,70$ \\
\hline Albumina $(\mathrm{g} / \mathrm{dL})$ & $4,53 \pm 0,50$ & $4,70 \pm 0,42$ & $1,5-4,10$ \\
\hline Globulina (g/dL) & $1,88 \pm 0,18$ & $1,95 \pm 0,21$ & $1,5-4,90$ \\
\hline $\mathrm{A}: \mathrm{G}$ & $2,42 \pm 0,18$ & $2,44 \pm 0,32$ & \\
\hline Ácido úrico ${ }^{*}$ & $0,66(0,0 ; 0,0)^{\mathrm{b}}$ & $0,89(0,9 ; 1,3)^{\mathrm{a}}$ & $0-2,857$ \\
\hline Ureia $(\mathrm{mg} / \mathrm{dL})$ & $31,01 \pm 6,70$ & $33,83 \pm 8,45$ & $5,04-24,36$ \\
\hline Creatinina (mg/dL) & $1,21 \pm 0,55$ & $1,81 \pm 0,60$ & $0,89-2,77$ \\
\hline Frutosamina (mg/dL) & $250,21 \pm 25,13$ & $245,85 \pm 47,82$ & --- \\
\hline \multicolumn{4}{|c|}{ Perfil Energético } \\
\hline Colesterol total (mg/dL) & $41,80 \pm 9,80$ & $41,63 \pm 12,33$ & $35,95-124,50$ \\
\hline Triglicerídeos* (mg/dL) & $59,95(33,4 ; 29,9)$ & $61,01(112,4 ; 205,8)$ & $0-237,18$ \\
\hline $\mathrm{HDL}(\mathrm{mg} / \mathrm{dL})$ & $3,62 \pm 0,81$ & $3,38 \pm 0,78$ & ----- \\
\hline Lactato $^{*}(\mathrm{mg} / \mathrm{dL})$ & $20,08(10,1 ; 10,0)$ & $28,28(68,4 ; 94,5)$ & ----- \\
\hline \multicolumn{4}{|c|}{ Perfil Mineral } \\
\hline $\mathrm{Ca}(\mathrm{mg} / \mathrm{dL})$ & $10,43 \pm 1,04$ & $10,31 \pm 0,77$ & $9,73-13,145$ \\
\hline $\mathrm{P}(\mathrm{mg} / \mathrm{dL})$ & $6,19 \pm 0,33$ & $5,44 \pm 0,73$ & $2,883-10,757$ \\
\hline $\mathrm{Ca}: \mathrm{P}(\mathrm{mg} / \mathrm{dL})$ & $1,69 \pm 0,20$ & $1,93 \pm 0,33$ & $1,0-3,60$ \\
\hline $\mathrm{Mg}(\mathrm{mg} / \mathrm{dL})$ & $2,88 \pm 0,25$ & $2,72 \pm 0,19$ & ---- \\
\hline $\mathrm{Na}(\mathrm{mEq} / \mathrm{L})$ & $172,33 \pm 25,85$ & $172,71 \pm 26,41$ & $127-145$ \\
\hline $\mathrm{K}(\mathrm{mEq} / \mathrm{L})$ & $4,78 \pm 2,38$ & $4,62 \pm 1,14$ & $3,7-7,50$ \\
\hline $\mathrm{Cl}(\mathrm{mEq} / \mathrm{L})$ & $90,02 \pm 2,55$ & $90,36 \pm 5,79$ & ---- \\
\hline
\end{tabular}

*Variáveis com dados não paramétricos; **ISIS (2013).

Os valores do eritrograma se mostraram dentro da normalidade (ISIS, 2013). No entanto, quando comparados com outros estudos, foram inferiores aos de Heijden et al. (2003), Madella et al. (2006) e Quadros (2017), mas próximos aos obtidos por
Di-Chiacchio et al. (2014). Diferenças metodológicas, climáticas, nutricionais, estado fisiológico, idade e manejo de contenção podem ser responsáveis pela diferença de resultados (Corredor-Matus e Rodríguez-Pulido, 2010; Di- 
Chiacchio et al., 2014; Eberhardt et al., 2015; Quadros 2017; Velasquez et al., 2017). Anemia microcítica, associada com deficiência de ferro, foi registrada por Di-Chiacchio et al. (2014), em capivaras de vida livre. A época do ano pode influenciar a oferta de alimentos e influenciar os valores do hemograma (Quadros, 2017). Este estudo foi realizado em épocas de abundância e de boa qualidade de alimentos, o que pode ter se refletido nos resultados do hemograma.

Os parâmetros do eritrograma (Hb, Ht e VCM) apresentaram diferenças significativas em relação à área de estudo, o que pode ser atribuído a diferenças climáticas (Arouca et al., 2000; Madella et al., 2006; Corredor-Matus e Rodríguez-Pulido, 2010; Eberhardt et al., 2015; Quadros 2017). Além disso, o peso dos animais pode também ter influenciado as médias menores dos animais da área 2, uma vez que dos seis indivíduos dessa área, dois eram juvenis.

Os valores do leucograma se mostraram dentro da normalidade (ISIS, 2013), o que também foi reportado por Di-Chiacchio et al. (2014), mas diferiram dos assinalados por Madella et al. (2006) e Quadros (2017). De acordo com Madella et al. (2006), animais de vida livre estão mais expostos a antígenos ambientais, podendo, dessa forma, desenvolver um sistema imunológico diferenciado, e isso não deve ser desconsiderado. Houve influência da área de estudo e do sexo dos animais na contagem de eosinófilos, uma vez que os animais da área 2 apresentaram valores elevados e fora dos padrões de referência (ISIS, 2013), o que também foi registrado por Heijden et al. (2003) e DiChiacchio et al. (2014). Neste estudo, todos os animais estavam parasitados por carrapatos e/ou por parasitos gastrointestinais (dados não apresentados), o que pode ser a causa da eosinofilia. De acordo com Campbell e Ellis (2007 apud Di-Chiacchio et al., 2014) e Eberhardt et al. (2013 e 2015), a eosinofilia é uma resposta não específica associada com infecções/infestações parasitárias, hipersensibilidade/reações alérgicas, infecções fúngicas e tumores, podendo ocorrer como resposta ao estresse crônico e à restrição alimentar, o que não ocorre com outras espécies de mamíferos.

Em relação às plaquetas, o valor encontrado esteve dentro da normalidade para a espécie
(ISIS, 2013), não havendo influência de nenhuma das variáveis avaliadas. $\mathrm{O}$ único estudo que avaliou a plaquetometria de capivaras foi o de Corredor-Matus e Rodríguez-Pulido (2010), no qual o valor determinado foi similar ao deste trabalho. A maioria dos parâmetros de bioquímica sanguínea se manteve dentro dos valores de referência (ISIS, 2013), exceto albumina, ureia, FA, AST e Na. Em São Paulo, Di-Chiacchio et al. (2014) registraram valores de ALT, FA, creatinina e albumina mais baixos do que os do presente estudo; os valores de PT foram similares e GGT e ureia foram superiores aos do mencionado estudo. Os autores destacaram que a albuminemia e os valores de PT aumentados poderiam ser atribuídos à carência nutricional e ao parasitismo, o que não ocorreu neste trabalho. Os valores obtidos por Quadros (2017) foram inferiores aos deste estudo no que se refere à ALT, AST, FA, ureia, creatinina, PT e albumina. Segundo a autora, o condicionamento dos animais para entrada no brete reduz o estresse da contenção, influenciando os valores obtidos.

Em animais em cativeiro, subadultos e adultos, Corredor-Matus e Rodríguez-Pulido (2010) obtiveram valores superiores aos do presente estudo nos parâmetros de ALT, GGT, ácido úrico, triglicerídeos, e valores inferiores nos parâmetros de AST, ureia, creatinina, PT e albumina. O colesterol foi o único parâmentro que mostrou resultado invertido em relação ao presente trabalho: superior nos animais subadultos e inferior nos adultos. A diminuição nos valores de PT e albumina está associada com nutrição deficiente (Di-Chiacchio et al., 2014).

Houve influência da área de estudo nos valores da atividade enzimática (FA), perfil proteico (PT, albumina e creatinina), energético (lactato) e mineral ( $\mathrm{Mg}$ e $\mathrm{Na}$ ). As principais causas de aumento da FA são colestase, obstrução biliar, osteomalácia, hiperparatireoidismo, tumor ósseo, deficiência de vitamina $\mathrm{D}$, raquitismo, hiperadrenocorticismo, em animais jovens $\mathrm{e}$ fêmeas gestantes (Lopes et al., 2007). Animais jovens e fêmeas gestantes apresentam níveis relativamente aumentados: em animais jovens, o valor da FA pode ser de duas a três vezes maior em relação aos adultos e, em animais gestantes, os valores podem chegar a $300 \%$ mais altos devido à presença do metabólito na placenta (Lopes et al., 2007). O valor mais elevado foi 
observado nos animais da área 3, majoritariamente constituído por fêmeas adultas.

Animais adultos apresentam teores de proteínas (albumina, globulina e fibrinogênio) mais elevados (Lopes et al., 2007; Corredor-Matus e Rodríguez-Pulido, 2010), o que pode explicar os valores mais baixos nos animais da área 1 , que eram majoritariamente juvenis, enquanto os das áreas 2 e 3 eram principalmente adultos. Além da idade, uma maior atividade muscular, relacionada à atividade física antes da captura, pode também resultar em níveis elevados de PT (Corredor-Matus e Rodríguez-Pulido, 2010). Os resultados obtidos eram compatíveis com os valores de referência (ISIS, 2013) para PT, mas elevados para albumina. Embora as fêmeas tenham apresentado valores ligeiramente mais elevados para albumina, esse parâmetro não foi influenciado pelo sexo dos animais. Fatores climáticos podem interferir nos resultados relativos à atividade enzimática e ao perfil proteico de capivaras de vida livre (Eberhardt et al., 2015; Quadros, 2017), conforme observado neste estudo.

A creatinina esteve dentro dos valores de referência (ISIS, 2013), embora tenha apresentado variação influenciada pela área de estudo, com valor mais elevado nos animais da área 3. Como essa enzima é formada do metabolismo da creatina e da fosfocreatina muscular e seus níveis sanguíneos não são afetados pela dieta, pela idade ou pelo sexo, mas, geralmente, aumentam em virtude da ocorrência de atividade física e desidratação (Lopes et al., 2007), os animais da área 3 apresentaram maior estresse pós-captura, o que poderia explicar os valores mais elevados em relação aos das áreas 1 e 2. Os animais avaliados por Quadros (2017) apresentaram um valor mais baixo de creatina do que os deste estudo, provavelmente porque foram submetidos ao condicionamento prévio à captura.

Os valores do ácido úrico foram influenciados tanto pela área de estudo quanto pelo sexo dos animais, no entanto estiveram no padrão de referência da espécie (ISIS, 2013). Na maioria dos mamíferos, esse metabólito provém da dieta, como resultado da quebra de ácidos nucleicos, e seu valor aumenta em neoplasias de células sanguíneas, doenças do fígado, insuficiência renal, endocrinopatias, hipotireodismo, entre outros (Lopes et al., 2007). Dessa forma, sua interpretação fica limitada em virtude da falta de outros exames mais específicos.

O perfil mineral foi compatível com os valores de referência (ISIS, 2013), à exceção do $\mathrm{Na}$, que apresentou níveis elevados. Corredor-Matus e Rodríguez-Pulido (2010) relataram valores inferiores, tanto em animais adultos como em subadultos, na grande maioria dos parâmetros avaliados, com exceção do nível mais elevado de K. O Na é um metabólito de grande importância no organismo, uma vez que é o principal responsável por manter a osmolaridade do plasma. Sua concentração é regulada pelos rins, que mantêm os níveis séricos em uma faixa estreita de variação. Os níveis séricos de $\mathrm{Na}$ podem apresentar-se elevados se houver incremento desse mineral na dieta, desidratação ou consumo reduzido de água, além de oligúria (Lopes et al., 2007). A alteração observada nos animais do presente estudo poderia estar relacionada ao jejum hídrico pós-captura. Os níveis séricos de $\mathrm{Mg}$ estiveram dentro da normalidade (ISIS, 2013), embora estivessem reduzidos nos animais da área 3 . A concentração desse mineral no organismo é um reflexo direto do seu nível na alimentação.

\section{CONCLUSÕES}

Os parâmetros hematológicos e bioquímicos séricos determinados neste estudo são considerados como referência e atestam a saúde e o bom estado nutricional de populações de capivaras em vida livre, na Mata Atlântica e na Caatinga da região Nordeste do Brasil. Diferenças entre os dois biomas e o sexo dos animais influenciaram alguns dos parâmetros avaliados. As informações aportadas neste estudo pioneiro na região Nordeste contribuem para aumentar o conhecimento sobre a ecofisiologia e a conservação in situ de capivaras.

\section{AGRADECIMENTOS}

Parte deste estudo foi financiada pela Coordenação de Aperfeiçoamento de Pessoal de Nível Superior - Brasil (Capes) - Código de Financiamento 001. Os autores agradecem tanto aos gestores do Parque Estadual Dois Irmãos e da Estação Ecológica do Tapacurá quanto ao proprietário da Chácara Paraíso, pela autorização para o uso dos animais neste estudo. 


\section{REFERÊNCIAS}

AROUCA, M.E.; MIRANDA, L.B.; LOPES, R. et al. Valores hematológicos de capivaras (Hydrochoerus Hydrochaeris) criadas em cativeiro no município de Botucatu, SP. Ciênc. Rural, v.30, p.813-817, 2000.

CARVALHO, C.C.D.; RAMOS, J.A.C.; RAMEHDE-ALBUQUERQUE, L.C. et al. Perfil hematológico, bioquímico sérico, proteína $\mathrm{C}$ reativa e cortisol de ararajubas (Guaroba guarouba) mantidas em cativeiro. Pesqui. Vet. Bras., v.33, p.394-398, 2013.

CORREDOR-MATUS, J.R.; RODRIGUEZ-PULIDO J.A. Estudio del perfil hemático y metabólico de chigüiros (Hydrochoerus hydrochaeris) (Linnaeus, 1766) en confinamiento. Orinoquia, v.14, p.95-109, 2010.

DI-CHIACCHIO, R.M.G; PRIOSTE, F.E.S.; VANSTREELS, R.E.T. et al. Health evaluation and survey of zoonotic pathogens in free-ranging capybaras (Hydrochoerus hydrochaeris). J. Wildl. Dis., v.50, p.496-504, 2014

EBERHARDT, A.T.; RUIZ, M.F.; BELDOMENICO, P.M.; RACCA, A.L. Dynamics of health of wild capybaras: biochemical and physiological parameters. Mammalia, v.80, p.413-423, 2015.

EBERHARDT, A.T.; BELDOMENICO, P.M.; MONJE, L.D.; RACCA, A.L. Biochemical and physiological parameters associated with Trypanosoma evansi prevalence in wild capybaras. Can. J. Zool., v.95, p.913-919, 2017.

FERRAZ, K.P.M.B.; VERDADE, L.M. Ecologia comportamental de capivaras: bases biológicas para o manejo da espécie. REUNIÃO SOCIEDADE. BRASILEIRA DE ZOOTECNIA, 36., 2001. Piracicaba. Anais.... Piracicaba, 2001. p.589-595.

HEIJDEN, K.M.; SZABÓ, M.P.J.; MATUSHIMA, E.R. et al. Valores hematológicos e identificação morfo-citoquímica de células sangüíneas de capivaras (Hydrochoerus hydrochoeris) parasitadas por carrapatos e capivaras livres de infestação. Acta Scient. Anim. Sci., v.25, p.143-150. 2003.
ISIS - International species information system (USA). Capybara reference ranges for physiological data values, 2013.

JIMÉNEZ, E.G. El capibara (Hydrochoerus hydrochaeris): estado actual de su producción. Roma: FAO, 1995.

MADELLA, D.A.; RODRIGUES NETO, E.J.; FELISBERTO, M.E.; SOUZA, C.E. Valores hematológicos de capivaras (Hydrochoerus hydrochaeris) (Rodentia: Hydrochoeridae) de vida livre na região de Campinas-SP. Ciênc. Rural, v.36, p.1321-1324, 2006.

MUÑOZ, K.D.; MONTOYA, E.G. Valores hemáticos del roncoso (Hydrochaeris hydrochaeris) en cautiveiro en la Amazonía Peruana. Rev. Investig. Vet. Perú, v.12, p.63-70, 2001

NOGUEIRA, S.S.C.; NOGUERIA-FILHO, S.L.G. Manual de criação de capivara. Viçosa: Centro de Produções Técnicas, 1996. 32p.

QUADROS, A.P.N. Parâmetros hematológicos $e$ bioquímicos de capivaras (Hydrochoerus hydrochaeris) do Distrito Federal. 2017. $27 \mathrm{f}$. Monografia (Graduação em Medicina Veterinária) Universidade de Brasília, DF.

SAS user's guide: Statics Version, Cary: SAS, 2009.

TRUPPEL J.H. Avaliação do parasitismo em capivaras (Hydrochaeris hydrochaeris) e sua atuação como hospedeiro intermediário de Neospora caninum e Toxoplasma gondii. Dissertação (Mestrado) Universidade Federal do Paraná, Curitiba, PR.

VELASQUEZ, J.C.C.; PACHECO, J.C.; HERNÁNDEZ, V.G.P. et al. Hematologia e bioquímica sanguínea da capivara (Hydrochoerus isthmius Goldman, 1912) no Departamento de Córdoba, Colômbia. In: CONGRESSO BRASILEIRO DA ANCLIVEPA, 2017, Olinda. Anais... Olinda: [s.n.], 2017. (Resumo). 